\title{
REGULARITY OF THE DISTANCE FUNCTION
}

\author{
ROBERT L. FOOTE
}

\begin{abstract}
A coordinate-free proof is given of the fact that the distance function $\delta$ for a $C^{k}$ submanifold $M$ of $\mathbf{R}^{n}$ is $C^{k}$ near $M$ when $k \geq 2$. The result holds also when $k=1$ if $M$ has a neighborhood with the unique nearest point property. The differentiability of $\delta$ in the $C^{1}$ case is seen to follow directly from geometric considerations.
\end{abstract}

In the study of analysis and geometry, the function that measures the distance to a submanifold plays an important role. Let $M$ be a submanifold of $\mathbf{R}^{n}$, and let $\delta: \mathbf{R}^{n} \rightarrow \mathbf{R}$ be the distance function for $M, \delta(x)=\operatorname{dist}(x, M)$. If $M$ is $C^{k}$, then $\delta$ is easily seen to be $C^{k-1}$ near $M$, since $\delta$ is always continuous and can be written in terms of the directions normal to $M$. It is the case, however, that $\delta$ is actually $C^{k}$ near $M$ when $k \geq 2$, and even when $k=1$ under certain circumstances. As Krantz and Parks [4] point out, this fact deserves to be better known that it is.

The regularity of $\delta$ was first considered in $[\mathbf{1}]$, and the proof for the case $k \geq 2$ is found in [2]. The combined results (including the case $k=1$ ) are given in [4] in a proof based on the work in $[\mathbf{1}]$.

The purpose of this note is to present a simple, coordinate-free proof of the following theorem and its $C^{1}$ analog.

THEOREM 1. Let $M \subset \mathbf{R}^{n}$ be a compact, $C^{k}$ submanifold with $k \geq 2$. Then $M$ has a neighborhood $U$ so that $\delta$ is $C^{k}$ on $U-M$.

In the $C^{1}$ case, the additional hypothesis is needed that some neighborhood of $M$ have the unique nearest point property. (See $[\mathbf{1}, \mathbf{4}]$.) A neighborhood $U$ of $M$ has this property if for every $x \in U$ there is a unique point $P(x) \in M$ so that $\delta(x)=\operatorname{dist}(x, P(x))$. The map $P: U \rightarrow M$ is called the projection onto $M$.

LEMMA. Let $M$ satisfy the hypothesis of Theorem 1. Then $M$ has a neighborhood $U$ with the unique nearest point property, and the projection map $P: U \rightarrow M$ is $C^{k-1}$.

PROOF. This is just the tubular neighborhood theorem with the added observation that the projection $P$ factors through the map that creates the neighborhood. (See $[\mathbf{3}]$.)

Let

$$
\nu(M)=\left\{(p, v) \in \mathbf{R}^{n} \times \mathbf{R}^{n} \mid p^{\prime} \in M \text { and } v \perp T_{p} M\right\}
$$

be the normal bundle for $M$; it is a $C^{k-1}$ manifold of dimension $n$. Define the $C^{k-1} \operatorname{map} F: \nu(M) \rightarrow \mathbf{R}^{n}$ by $F(p, v)=p+v$. The Jacobian $F_{*}$ is easily seen to be nonsingular along the zero section $\{(p, 0) \in \nu(M)\}$. By the inverse function theorem and the compactness of $M$, there is an $\varepsilon>0$ such that $F$ restricted to

Received by the editors September 26, 1983.

1980 Mathematics Subject Classification. Primary 53A07; Secondary 26B05.

(C) 1984 American Mathematical Society $0002-9939 / 84 \$ 1.00+\$ .25$ per page 
$\nu_{\varepsilon}(M)=\{(p, v) \in \nu(M):|v|<\varepsilon\}$ is a $C^{k-1}$ diffeomorphism onto a neighborhood $U$ of $M$. On $U$ the map $P$ is the composition

$$
U \stackrel{F^{-1}}{\rightarrow} \nu_{\varepsilon}(M) \rightarrow M
$$

where the last map is projection onto the first factor. Q.E.D.

In [4] $M$ is said to have positive reach of at least $\varepsilon$. The largest possible neighborhood $U$ on which $P$ is defined is determined in part by the local extrinsic geometry of $M$ inside $\mathbf{R}^{n}$ : the extrinsic curvature of $M$ governs the location of the singularities of the map $F: \nu(M) \rightarrow \mathbf{R}^{n}$. (See $[5, \S 6]$.)

Proof OF ThEOREM 1. On the neighborhood $U$ where $P$ is well defined, the distance function is given by $\delta(x)=\|x-P(x)\|$. For $v \in \mathbf{R}^{n}$, let $D_{v}$ denote differentiation in the direction $v$. Then for $x \in U-M$,

$$
\left(D_{v} \delta^{2}\right)(x)=2(x-P(x)) \cdot\left(v-D_{v} P(x)\right)=2(x-P(x)) \cdot v,
$$

since $D_{v} P(x)$ is tangent to $M$. Hence

$$
\left(\operatorname{grad} \delta^{2}\right)(x)=2(x-P(x)),
$$

which is $C^{k-1}$, and so $\delta$ is $C^{k}$ on $U-M$. Q.E.D.

In the $C^{1}$ case one needs to examine the behavior of the difference quotient.

THEOREM 2. Let $M$ be $C^{1}$ and suppose $U$ is a neighborhood of $M$ with the unique nearest point property. Then $\delta$ is $C^{1}$ on $U-M$.

ProOF. A simple argument (see $[\mathbf{1}, 4.8(4)]$ ) shows that $P: U \rightarrow M$ is continuous. Thus, it suffices to show that $(*)$ holds on $U-M$. If this is not the case, then there is some point $x \in U-M$ and some vector $v \in \mathbf{R}^{n}$ such that

$$
\varliminf_{t \rightarrow 0^{+}} \frac{\delta^{2}(x+t v)-\delta^{2}(x)}{t}<2(x-P(x)) \cdot v
$$

or

$$
\varlimsup_{t \rightarrow 0^{+}} \frac{\delta^{2}(x+t v)-\delta^{2}(x)}{t}>2(x-P(x)) \cdot v .
$$

In the first case, one can find a fixed $\varepsilon>0$ and then choose $t>0$ arbitrarily close to zero such that

$$
\delta^{2}(x+t v)<\delta^{2}(x)+2(x-P(x)) \cdot t v-t \varepsilon .
$$

It follows, then, that

$$
\begin{aligned}
\operatorname{dist}^{2}(x, P(x+t v)) & =\|(x+t v-P(x+t v))-t v\|^{2} \\
& =\delta^{2}(x+t v)-2(x+t v-P(x+t v)) \cdot t v+t^{2}\|v\|^{2} \\
& <\delta^{2}(x)+2(P(x+t v)-P(x)) \cdot t v-t \varepsilon-t^{2}\|v\|^{2} .
\end{aligned}
$$

By the continuity of $P, t$ can be chosen small enough so that

$$
\operatorname{dist}(x, P(x+t v))<\delta(x)=\operatorname{dist}(x, P(x)) .
$$

Then $x$ is closer to $P(x+t v)$ than to $P(x)$, a contradiction.

Similarly, (2) leads to $\operatorname{dist}(x+t v, P(x))<\operatorname{dist}(x+t v, P(x+t v))$. The theorem follows. Q.E.D. 
REMARKS. (1) With some modifications, the same proofs will work when $M$ is a submanifold of a Riemannian manifold.

(2) If $M$ is a hypersurface of the form $M=\left\{x \in \mathbf{R}^{n} \mid \rho(x)=0\right\}$, where $\rho$ is a $C^{k}$ function with $d \rho \neq 0$ on $M$, then one can form the signed distance function

$$
\tilde{\delta}(x)=\left\{\begin{array}{cc}
\delta(x) & \text { for } \rho(x) \geq 0 \\
-\delta(x) & \text { for } \rho(x) \leq 0
\end{array}\right.
$$

It is easy to see that $\tilde{\delta}$ is $C^{k}$ on all of $U$.

(3) In the $C^{1}$ case, the regularity of $M$ does not enter into the proof. $M$ can be replaced by any closed set in $\mathbf{R}^{n}$, and $U$ by any open set on which the projection $P: U \rightarrow M$ is well defined. (For the original treatment of this, see [1].)

(4) The extra hypothesis in the $C^{1}$ case is essential. The distance function for the curve $y=|x|^{3 / 2}$ in $\mathbf{R}^{2}$ is not differentiable at any point on the $y$-axis. See [4] for details.

For further remarks and examples, the reader is directed to the references, especially $[\mathbf{4}]$.

\section{REFERENCES}

1. H. Federer, Curvature measures, Trans. Amer. Math. Soc. 93 (1959), 418-491.

2. D. Gilbarg and N. Trudinger, Elliptic partial differential equations of second order, Springer-Verlag, Berlin, 1977.

3. V. Guillemin and A. Pollack, Differential topology, Prentice-Hall, Englewood Cliffs, N.J., 1974.

4. S. Krantz and H. Parks, Distance to $C^{k}$ hypersurfaces, J. Differential Equations 40 (1981), 116-120.

5. J. Milnor, Morse theory, Princeton Univ. Press, Princeton, N.J., 1963.

Department of Mathematics, TeXas TeCH University, LubBock, TeXas 79409 